\title{
A visually induced illusion of body tilt in a horizontal plane
}

\author{
DONALD R. GOODENOUGH, ANDRZEJ NOWAK, PHILIP K. OLTMAN, \\ PATRICIA W. COX, and ERIC SIGMAN \\ Educational Testing Service, Princeton, New Jersey 08541
}

\begin{abstract}
Unexpected spontaneous reports of an illusion of body orientation have been observed in previous studies on the effects of a tilted overhead frame on supine observers. The present studies were designed in an attempt to describe and measure this illusion. The findings suggest that when objectively aligned with invisible side walls of the room, some subjects may experience an illusion that they are tilted in a horizontal plane with respect to the room walls in the direction opposite to frame tilt from the longitudinal body axes. The results are discussed in terms of a model of visual-vestibular interactions.
\end{abstract}

When upright observers view a retinally large square frame that is tilted in a frontal plane, they behave in a variety of ways as if their bodies were tilted in the opposite direction. If normal subjects are tilted in the dark in a clockwise $(\mathrm{CW})$ direction, for example, they report a feeling of CW self-tilt; settings of a visible rod to alignment with the perceived vertical are rotated from the longitudinal body axis in a counterclockwise (CCW) direction, and the eyes rotate countertorsionally in a $\mathrm{CCW}$ direction, coming to rest at a less tilted position than the head (e.g., Howard \& Templeton, 1966). The same responses can also be produced in upright observers by a frame that is tilted $\mathrm{CCW}$. The most widely studied of these responses involves vertical rod settings, often used to measure individual differences in degree of perceptual field dependence (e.g., Witkin \& Asch, 1948; Witkin, Dyk, Faterson, Goodenough, \& Karp, 1962/1974). When a rod is surrounded by a $\mathrm{CCW}$ frame, vertical settings tend to err in a $\mathrm{CCW}$ direction. Under these conditions, subjects may also experience an illusion of CW self-tilt (Ebenholtz \& Benzchawel, 1977; Sigman, Goodenough, \& Flannagan, 1978,1979 ), and a torsional rotation of the eyes in the CCW direction of frame tilt also occurs (Goodenough, Oltman, Sigman, \& Cox, 1981; Goodenough, Sigman, Oltman, Rosso, \& Mertz, 1979; Hughes, 1973).

Some similar responses have also been recorded in supine observers while viewing an overhead frame that is tilted in a horizontal plane from the longitudinal axis of the body. Research on overhead displays has been motivated by the question of whether gravitational cues moderate visual effects on tilt per-

These studies were conducted with the aid of NIMH Grant 21989. Requests for reprints should be directed to Donald $R$. Goodenough, Educational Testing Service, Princeton, New Jersey 08541 . ception. Since the gravitational direction is irrelevant to the definition of tilt in horizontal planes for a supine observer, the usual instructions for vertical rod settings cannot be used under these conditions. Subjects have therefore been asked to align the rod with the body (Brosgole \& Cristal, 1967; Goodenough et al., 1981; Templeton, 1973) or with an invisible wall of the laboratory room that is parallel with the longitudinal body axis (Lichtenstein \& Saucer, 1974). Rod-adjustment errors in the direction of frame tilt have been found under these conditions. Visually induced eye torsion toward the axes of the frame has also been recorded in supine observers (Goodenough et al., 1981). These frame effects on rod adjustments and eye torsion are in the same direction as, and may even be larger than, they are in upright observers, suggesting that gravitational cues play some moderating role when relevant to tilt perception.

The studies described below are concerned with another similarity in frame effects between the upright and supine body positions. They were suggested by unsolicited reports of an illusion of self-tilt given by some supine observers of overhead displays in previous studies from our laboratory (Goodenough et al., 1981). While viewing the tilted square in an otherwise dark room, a few of our subjects claimed that their bodies felt tilted in a horizontal plane with respect to the unseen walls of the laboratory. Although they knew that they were lying parallel to the side walls, with their feet pointing toward an end wall, they reported feeling that their bodies had rotated in the direction opposite to the frame when it was turned on, so that their feet seemed to be pointing toward a corner of the room.

These observations were unexpected. Like the illusory experience of body orientation noted by our supine observers, the self-tilt illusion found in erect observers might be describable with reference to the unseen walls of the laboratory room. Since body tilt 
from the erect position is normally defined with respect to the gravitational vertical, however, it had not occurred to us to look for an illusion of selftilt in the supine position until our subjects called this possibility to our attention.

Following our subjects' lead, we first undertook some exploratory work using the authors as observers, and we also could sometimes report a compelling illusion. While the conditions required to produce the feeling of self-tilt could not be clearly identified, the exploratory work did suggest that the illusion might be facilitated if subjects were given an opportunity to study the location of environmental landmarks while the room was lit and were encouraged to retain a cognitive map or image of the laboratory after the room lights were turned off. Indeed, it is not clear to us whether observers can be asked a nontrivial question about feelings of self-tilt in a horizontal plane in the absence of any knowledge about environmental axes other than the immediately perceived angle between the frame and the body.

More systematic studies were then conducted in the attempt to document the illusion. In the first experiment, designed primarily as a pilot study for a different purpose, ${ }^{1}$ supine subjects were routinely asked to describe their feelings of self-orientation in the room. In the second experiment, an attempt was also made to develop an objective method of measuring illusion magnitude in the upright and supine positions.

\section{EXPERIMENT 1}

\section{Method}

Twelve paid volunteers were told that a few subjects in previous studies had mentioned a change in their sense of body orientation while looking at the frame and that one of the study goals was to see whether, and under what conditions, any such change might occur. They were not told the kind or direction of illusion that might be expected.

After the procedures were explained, the subjects were asked to lie on their backs on a padded table to which clamps were attached for holding the head in a fixed position with the trunk approximately aligned with the head and the side walls of the room. They were given an opportunity to study their orientation in the room with the lights on before each trial.

At the start of each trial, the room lights were turned off and the electroluminescent frame was turned on. The frame was located $112 \mathrm{~cm}$ above the subject's head, subtending $28 \mathrm{deg}$ of visual angle per side. It was rotatable around its center, located on a vertical axis that intersected the subject's mid-eye position. The equipment is described elsewhere in greater detail (Goodenough et al., 1981).

Three trials were given to each subject. Each trial lasted for about a minute, during which the subjects were asked to look at the frame and after which they were asked to describe any change in perceived body orientation. During the first and third of these trials, the frame sides were set parallel with the longitudinal body axis. During the second trial, the frame was tilted by $22.5 \mathrm{deg} \mathrm{CW}$ for a randomly selected half of the subjects and $22.5 \mathrm{deg}$ CCW for the other half.

\section{Results}

Six of the 12 subjects reported that their bodies felt tilted in the room during the second, frame-tilted trial in the supine position. In all six of these cases, the self-tilt illusion occurred in a horizontal plane and in the direction opposite to the frame tilt. None of the subjects reported an experience of self-tilt during the first or third trials when the frame was aligned with the body.

\section{EXPERIMENT 2}

While the data from the first experiment provided some support for the hypothesis that a visually induced illusion of self-tilt occurs in some supine observers, there are some obvious problems with the method of subjective report. Many subjects are not very articulate in describing their experiences, and it is hard to measure illusion magnitude even among subjects who are. A more objective method of documenting and measuring the illusion would clearly be desirable.

With frames that are tilted in a vertical plane, effects on perceived self-orientation have been measured most directly by asking observers to adjust their bodies to the gravitational vertical (e.g., Howard \& Templeton, 1966). It may also be possible to measure perceived body tilt less directly, however, by comparing rod settings to the vertical and the head. When the observer is objectively upright, the gravitational vertical coincides with the longitudinal head axis. It seems reasonable to assume, therefore, that when an observer feels upright, a rod that looks aligned with the head should also look aligned with the gravitational vertical. Thus, one might expect no difference between rod settings to the head and to the gravitational vertical in the absence of illusory self-tilt. To the extent that the observer feels tilted, however, one might expect a displacement of vertical settings from head settings in the direction opposite to perceived body tilt. Differences of this sort have been found in the standard rod-andframe test under some conditions, consistent with body-adjustment measures of illusory self-tilt (Sigman et al., 1979).

It is not obvious how the body-adjustment method could be used to provide equivalent measures of perceived self-orientation in the erect and supine positions. The rod-adjustment method can be adapted, however, by substituting instructions to align the rod with a side wall of the room for the usual vertical alignment instructions. This rod-adjustment method was used in the second experiment.

More specifically, we hypothesized that rodadjustment errors in the direction of frame tilt would be greater under instructions to align the rod with the wall than with the head, and that the instructional difference would be greater in the supine than in the upright position and greater among subjects who reported the self-tilt illusion than among subjects who did not. 
Since frame effects in upright observers are directly related to the retinal size of the display (Ebenholtz, 1977; Ebenholtz \& Benzschawel, 1977; Ebenholtz \& Callan, 1980), it seemed reasonable to hypothesize that the supine self-tilt illusion would also vary with retinal size.

\section{Method}

The subjects were 18 paid volunteers. The standard rod and frame described in Experiment 1 was used for 8 of these subjects (frame sides subtending $28 \mathrm{deg}$ of visual angle), and the display was moved closer to the other 10 subjects, so that the sides subtended $40 \mathrm{deg}$ of visual angle. All subjects were run in both the upright and supine body positions, with an upright-supine sequence for half of the subjects in each frame-size group and a supine-upright sequence used for the remainder.

There were three frame conditions in each body position: frame absent, $22.5 \mathrm{deg} \mathrm{CW}$ and $22.5 \mathrm{deg} \mathrm{CCW}$. The sequence of conditions was absent-CW-CCW for half of the subjects and absent$\mathrm{CCW}-\mathrm{CW}$ for the other half. Four rod adjustments were made during each frame condition, consisting of all combinations of two alignment instructions (head, wall) and two rod starting positions (22.5 deg CW, $22.5 \mathrm{deg} \mathrm{CCW}$ ) given in counterbalanced sequences across subjects. The subjects were asked to describe their body orientation in the room after each rod adjustment.

The equipment and procedures were otherwise the same as in the first experiment.

\section{Results}

The algebraic means of the rod-adjustment errors, signed in the direction of frame tilt, are shown in Table $1 .^{2}$ Analyses of variance on these data show significant main effects of instruction $[F(1,16)=17.49$, $\mathrm{p}<.001]$ and body position $[\mathrm{F}(1,16)=15.64, \mathrm{p}<.01]$ and a significant instruction $\times$ body position interaction $[F(1,16)=11.45, p<.01]$. The interaction is due to the fact that errors in the direction of the tilted frame are much greater for wall than for head settings in the supine position, but not much different in the upright position. Head and wall settings differ significantly in the supine position $[F(1,16)=$ $18.17, \mathrm{p}<.001$ ], but not in the upright position. The large frame tended to produce slightly larger errors in wall settings than the small frame, but this difference is insignificant.

Table 1

Means and Standard Errors of Means for Rod-Adjustment Errors (in Degrees) in the Direction of Frame Tilt

\begin{tabular}{ccccc}
\hline & \multicolumn{4}{c}{ Instructions } \\
\cline { 2 - 5 } Frame & \multicolumn{2}{c}{ Head } & \multicolumn{2}{c}{ Wall } \\
\cline { 2 - 5 } \cline { 3 - 4 } Size & Mean & SEM & Mean & SEM \\
\hline & \multicolumn{4}{c}{ Upright Body Position } \\
Small & 4.5 & .7 & 4.7 & 1.1 \\
Large & 4.2 & .8 & 5.1 & 1.6 \\
Total & 4.3 & .5 & 4.9 & 1.0 \\
& \multicolumn{4}{c}{ Supine Body Position } \\
Small & 7.1 & 1.3 & 9.5 & \\
Large & 5.3 & 1.3 & 12.3 & 2.5 \\
Total & 6.1 & .9 & 11.1 & 1.3 \\
\hline
\end{tabular}

The data on subjective reports are not quite as clear as in the first experiment, perhaps due to occasional experiences of body motion and/or tilt in response to rotations of the rod during the adjustment procedure. A few reports of changes in body orientation were given, even in the rod-alone condition. In the supine position, however, reports of selftilt were predominantly opposite in direction to the tilted frame. One subject reported sensations of selftilt in the unexpected direction after one rod adjustment during one frame-tilted trial but consistently felt tilted away from the frame during the other trial. In seven other subjects, reports of self-tilt in the direction opposite to the frame were given on one or both frame-tilted trials. One of these seven cases consistently reported a similar feeling of self-tilt during the rod-alone trial, perhaps due to a slight objective body misalignment in the room. Excluding this case, five of the self-tilt reports were given in the large-frame condition and two with the small frame. Reports of body tilt were also given by five of the subjects in the upright position, but three of these were in the direction of frame tilt. Thus, no evidence could be found of the expected self-tilt illusion in upright observers.

Among the seven subjects whose subjective reports did suggest a supine self-tilt illusion, wallsetting errors exceeded head-setting errors by a mean of $7.9 \mathrm{deg}$. Among the remaining 11 subjects, the wall-head difference was smaller, but in the same direction (mean difference $=3.1 \mathrm{deg}$ ). Among the subjects who did not report a supine self-tilt illusion, the difference was due largely to one outlying subject whose wall errors were very large. The difference scores were therefore examined by the nonparametric median test. For this purpose, the subjects were divided into upper and lower halves of their frame-size groups on the basis of their headwall difference scores, with the result that six of the seven subjects who evidently experienced the illusion, but only three of the 11 subjects who did not, were located above the median split $(p<.05)$. These results are consistent with the view that the rodadjustment method may provide a useful index of the supine self-tilt illusion.

\section{DISCUSSION}

The occurrence of a supine self-tilt illusion may have relevance for some practical, as well as some theoretical, issues. From a practical perspective, the experiences reported by our subjects may be related to problems of orientation perception under zerogravity conditions. As is true for the perception of tilt in a horizontal plane on earth, no relevant gravitational information is available for tilt perception when observers are stationary in outer space. Never- 
theless, disturbing experiences of self-tilt have been reported anecdotally by some astronauts when misaligned with the visible walls of their spacecraft (e.g., Cooper, 1976). The illusion of self-tilt described by some of our subjects may be due to a similar phenomenon. If so, then further studies of the supine self-tilt illusion may aid in the attempt to understand orientation disturbances in space flight.

From a theoretical perspective, the occurrence of a self-tilt experience suggests that the effects of a tilted overhead frame may not be attributable entirely to intravisual factors. As in the upright position, supine observers seem to respond as if they were objectively tilted in the opposite direction with respect to environmental axes. In upright observers, it has been suggested that these responses are mediated by visualvestibular interactions (e.g., Bischof, 1974). In this view, responses that occur when the otolith is stimulated by objective body tilts are also induced in upright observers by peripheral retinal configurations that normally occur when the body is tilted. If this view is correct, then the fact that visually induced eye torsion occurs in supine as well as in upright observers (Goodenough et al., 1981) should not be surprising, and a visually induced sensation of selftilt might also be expected, whether or not the gravitational direction is relevant. Indeed, it is not uncommon for supine observers to report feelings of "dizziness," "floating," "bending," and so on, while watching the tilted frame, perhaps reflecting a visually induced sensation from the vestibular system that is cognitively not very articulate in the absence of well-defined environmental axes for use in interpreting the sensation perceptually. Nor should it be too surprising to find that frames that are tilted in a frontal plane induce larger responses of the otolith system in the supine than in the upright condition. It has been suggested that visual effects on otolithsystem responses increase under conditions in which utricular sensitivity decreases (Bischof, 1974). Since the utricles are sensitive to differences in body tilt from the environmental axes in a vertical plane but not in a horizontal plane, they may serve to moderate visual effects on lateral tilt perception in the upright but not the supine position. Thus, both the occurrence of a supine self-tilt illusion and the difference in illusion magnitude between upright and supine observers appear to be consistent with a model of visual-vestibular interactions.

While the self-tilt illusion showed some tendency in our data to be greater when the visual angle subtended by the frame was larger, this hypothesized effect was insignificant. Since frame size was introduced as a between-subjects factor in the design, the failure to find a significant effect might be attributable to the great variability among subjects. In any case, it seems clear from previous studies that frame effects on tilt perception do vary directly with the retinal size of the display (e.g., Ebenholtz, 1977). These findings may be due to the fact that a frame that is larger (or closer to the observer) impinges more on the retinal periphery (Ebenholtz \& Callan, 1980). The effects of retinal eccentricity have been studied extensively in the case of visual-vestibular interactions involving moving visual stimuli. For example, a rotating visual field is capable of inducing illusions of self-rotation in the opposite direction (e.g., Dichgans \& Brandt, 1974; Finke \& Held, 1978), evidently due to visual driving of the vestibular system. ${ }^{3}$ To be effective, however, the stimulus must impinge upon the peripheral retinal system (Dichgans \& Brandt, 1974). It seems reasonable to suppose that illusions of self-tilt that can be induced by a stationary visual stimulus, as in the present experiments, are analogous to the more widely studied illusions of self-rotation induced by moving visual stimuli. If so, then the effects of frame size may be attributable to the role of the peripheral retinal system in the mediation of visual-vestibular interactions involving stationary or moving displays.

While a model of visual-vestibular interactions appears useful in the attempt to understand the selftilt experience, a number of unanswered questions remain. It is not clear why reports of visually induced changes in perceived body orientation have not been reported by all observers or even all the time in the same observers under similar conditions. It is interesting to note that variability in observed phenomena due to individual differences, habituation, and so on, is often emphasized in the literature on visualvestibular interactions (e.g., Bischof, 1974). But the problem remains of identifying the stimulus conditions that are necessary and sufficient to produce the self-tilt illusion more specifically. The rod-adjustment method used in the second experiment may aid in the solution.

\section{REFERENCES}

Bischof, N. Optic-vestibular orientation to the vertical. In H. H. Kornhuber (Ed.), Handbook of sensory physiology: Vestibular system (Pt. 2). New York: Springer-Verlag, 1974.

Brosgole, L., \& Cristal, R. M. The role of phenomenal displacement on the perception of the visual upright. Perception \& Psychophysics, 1967, 2, 179-188.

Cooper, H. S. F., JR. A reporter at large: Life in a space station. The New Yorker, August 30, 1976, pp. 34-66, and September 6, 1976, pp. 34-70.

Daunton, N. G., \& Thomsen, D. D. Otolith-visual interactions in single units of cat vestibular nuclei. Neuroscience Abstracts, $1976,2,1057$.

Dichgans, J., \& Brandt, T. The psychophysics of visually induced perception of self-motion and tilt. In F. O. Schmitt \& F. G. Worden (Eds.), The neurosciences: Third study program. Cambridge, Mass: M.I.T. Press, 1974.

Ebenholtz, S. M. Determinants of the rod and frame effect: The role of retinal size. Perception \& Psychophysics, 1977, 22, 531-538. 
Ebenholtz, S. M., \& Benzschawel, T. L. The rod and frame effect and induced head tilt as a function of observation distance. Perception \& Psychophysics, 1977, 22, 491-496.

Ebenholtz, S. M., \& Callan, J. W. Modulation of the rod and frame effect: Retinal angle vs, apparent size. Psychological Research, 1980, 42, 327-334.

FinKe, R. A., \& HeLd, R. State reversals of optically induced tilt and torsional eye movements. Perception \& Psychophysics, $1978,23,337-340$.

Goodenough, D. R., Oltman, P. K., Sigman, E., \& Cox, P. W. The rod-and-frame illusion in erect and supine observers. Perception \& Psychophysics, 1981, 29, 365-370.

Goodenough, D. R., Sigman, E., Oltman, P. K., Rosso, J., \& Mertz, H. Eye torsion in response to a tilted visual stimulus. Vision Research, 1979, 19, 1177-1179.

Henn, V., Young, L. R., \& Finley, C. Vestibular nucleus units in alert monkeys are also influenced by moving visual fields. Brain Research, 1974, 71, 144-149.

How ARD, I. P., \& TEMPLETON, W. B. Human spatial orientation. Toronto: Wiley, 1966.

Hughes, P. C. The influence of the visual field upon the visual vertical in relation to ocular torsion of the eye (Doctoral dissertation, University of Oklahoma, 1973). Dissertation Abstracts International, 1973, 33, 4686B. (University Microfilms No. 739158)

Lichtenstein, J. H., \& Saucer, R. T. Visual dependency in the erect and supine positions. Journal of Applied Psychology, $1974,59,529-531$.

Sigman, E., Goodenough, D. R., \& Flannagan, M. Subjective estimates of body tilt and the rod-and-frame test. Perceptual and Motor Skills, 1978, 47, 1051-1056.

Sigman, E., Goodenough, D. R., \& Flannagan, M. Instruc- tions, illusory self-tilt, and the rod-and-frame test. Quarterly Journal of Experimental Psychology, 1979, 31, 155-165.

TEMPLETon, W. B. The role of gravitational cues in the judgment of visual orientation. Perception \& Psychophysics, 1973, 14, 451-457.

Witkin, H. A., \& Asch, S. E. Studies in space orientation: IV. Further experiments on perception of the upright with displaced visual fields. Journal of Experimental Psychology, 1948, 38, 762-782.

Witkin, H. A., Dyk, R. B., Faterson, H. F., Goodenough, D. R., \& Karp, S. A. Psychological differentiation. Potomac, Md: Erlbaum, 1974. (Originally published, Wiley, 1962.)

\section{NOTES}

1. This study was designed primarily for the purpose of exploring frame effects on auditory localization. Accordingly, the subjects were also asked to adjust a click sound source under various conditions while viewing the display.

2 . Errors in setting the rod to the wall were significantly greater $[F(1,16)=5.46, p<.05]$ when viewing the $\mathrm{CCW}$ frame (mean $=$ $+9.4 \mathrm{deg}$ ) than when viewing the $\mathrm{CW}$ frame (mean $=+6.5 \mathrm{deg}$ ). No significant difference in magnitude was found between $\mathrm{CW}$ and CCW frame effects on head settings.

3. Responses of the vestibular system to moving visual stimuli have also been recorded in animals from single cells at the level of the vestibular nuclei (e.g., Daunton \& Thomsen, 1976; Henn, Young, \& Finley, 1974).

(Manuscript received August 20, 1981; revision accepted for publication November 25,1981 .) 